\title{
Fractional models of seismoacoustic and electromagnetic activity
}

\author{
Boris Shevtsov ${ }^{1, \star}$ and Olga Sheremetyeva ${ }^{1,2, \star \star}$ \\ ${ }^{1}$ Institute of Cosmophysical Research and Radio Wave Propagation FEB RAS, Paratunka, Russia \\ ${ }^{2}$ Vitus Bering Kamchatka State University, Petropavlovsk-Kamchatskiy, Russia
}

\begin{abstract}
Statistical models of the seismoacoustic and electromagnetic activity caused by deformation disturbances are considered on the basis of compound Poisson process and its fractional generalizations. Wave representations of these processes are used too. It is discussed five regimes of deformation activity and their role in understanding of the earthquakes precursors nature.
\end{abstract}

\section{Introduction}

Modeling of various regimes of seismoacoustic and electromagnetic emission caused by deformation disturbances, especially in the phase of their activity, is actual for understanding of earthquakes precursors nature. The changes in the regimes of the deformation process are of interest: unexpected decreases and increases in their activity, the duration of fading, and the discharging energy of accumulated stresses. All these features are detected in signals of seismoacoustic and electromagnetic emission, which are used for diagnostics of the deformation process.

The work of external forces supports a critical level of elastic stresses, as a result there are constantly growing plastic deformations. This process is characterized by the velosity of random dislocation changes, which are determined by the parameters $L$ and $u$ of the dislocation tensor - the spatial scale and the discontinuity of displacement vector (the Burger's vector). We suppose other parameters of the tensor are unchanged, but it is not difficult to take into account the full tensor description.

Note that with using the theory of dislocation changes, it is possible to consider discontinuities, moving along existing faults, repacking of grains or blocks, filtration of liquid in a porous medium, etc. in a wide range of scales $L$ (up to 15 orders).

\section{Fractional model}

If the relative elastic deformations $\varepsilon$ are constant, then we can take the following $u / L=\varepsilon$. In this case there is can considered random dislocation changes as a one parameter process $L_{V, T}(t)=\sum_{k=0}^{N} L\left(x_{k}, t_{k}\right)$, where the sum is taken over the all dislocations with coordinates $x_{k}, t_{k}$ in a given volume $V$ and time interval $T$.

\footnotetext{
${ }^{\star}$ Corresponding author: bshev@ikir.ru

${ }^{\star \star}$ Corresponding author: olga.v.sheremetyeva@gmail.com
} 
Random variables are $x_{k}, t_{k}$ and $L\left(x_{k}, t_{k}\right)$. In the first approximation, they can be considered independent, then the process $L_{V, T}(t)=\sum_{k=0}^{N} L\left(x_{k}, t_{k}\right)$ is a compound Poisson process with the distribution of independent increments $p(L)$. For $N \rightarrow \infty$, we'll have a stable distribution $p\left(L_{V, T}, t\right)$ [8] with an increasing average that characterizes the process of plastic deformations in the volume $V$.

If the distribution of independent increments $p(L)$ is stable, then the distribution $p\left(L_{V, T}, t\right)$ will be stable at each step $N$ with time growing parameters. This process is generalization of the Gaussian process and is the key to understanding of the universality and wide prevalence in nature of stable distributions having the power law properties. The Gutenberg-Richter law can also be approximated by these distributions on the assumption that dislocation changes are independent.

Independence of random variables $x_{k}, t_{k}$ and $L\left(x_{k}, t_{k}\right)$ is violated in dislocation clusters, in which the dislocation density is so large that their radii of influence $R_{\text {inf }}$ and $T_{\text {inf }}$ depending on $L\left(x_{k}, t_{k}\right)$ are overlap [1,2]. About modeling of zones of influence see $[3,4]$. The difference between sparse and dense packing of dislocations in clusters may be distinguished.

Using the seismic process as an example we can considered the sequences of foreshocks and aftershocks as a sparse packing and the mainshock (fault) as a close packing of dislocations. The events cluster is a plastic deformation disturbance with a time growing packing of events. Obviously, that the dependence and connectedness of the events is increased with their density. The sequence of foreshocks can be considered as a phase of consolidation of certain scaled level events, which leads to the mainshock - event of more higher scaled level. A sequence of aftershocks can be regarded as the branching phase of the mainshock.

Such representation of the plastic deformation disturbance gives us the opportunity to see the interaction of scale levels. In the space time representation, the cluster of events is developed as a process of random walks that it is possible to compare with a fractional diffusion process [1,2], in general, non-Markovian, nonlocal and with memory. It means that in the sequence of events all random variables $L\left(x_{k}, t_{k}\right)$ and $x_{k}, t_{k}$ are dependent. Possible simplifications will be discussed below.

Influence radii $R_{\text {inf }}$ and $T_{\text {inf }}$ increase with $L\left(x_{k}, t_{k}\right)$ and become larger than the size of the area $(V, T)$, this leads to that the large events always form a cluster in the considerational area [1,2], so there are constraints to use the Poisson process for modelling large events.

For small events, there is an accumulating effect. Over time, the density of small dislocations increase, they consolidate and cause an event of more larger scale (mainshock), after which, as a rule, a backward wave arises down along a cascade of scales in the form of a branching process. With such cascade effects, events of different scales can not be considered independent.

Thus, we have the constraints on the use of the Poisson process as a model of dislocational deformations, both for large and small events. Nevertheless, for any of the chosen scales, assuming their equality, we can use the Poisson process as a first approximation or a background regime. Such events are referred as migrants for area $(V, T)$ [5]. And it is necessary to consider deviations from the background regime to take into account more subtle effects.

Deviation from the background regime can occur in the hardening or relieving (weakening) medium (changes in rheology). When the medium is hardening there is arose the area in which the process of plastic deformation slows down and the flow of events decreases. Thus, for example, there is an area of seismic quiescence or gap (Fedotov), in which deformation heterogeneity is formed. The elastic energy accumulated in it is released when the stresses are discharged.

It is clear that such accumulation of energy can not be described by the Poisson process with a flow of independent events, because there is a high degree of localization of events that did not release in a seismic quiescence, but they will be realized in deformation disturbance. They can be regarded as adjourned events. Deficiency of events leads to an increase in elastic stresses, as a result the strengthening of the medium is overcome and the accumulated additional elastic energy is released. When 
the medium is hardening the sequential changes in the regimes of the plastic deformation process take place: normal (background) pulsations and their deceleration (fading), then activation (discharge) and again the background regim. This sequence of changes is called intermittency. When the medium is weaken, the terms "deceleration" and "activation" should be replaced one to another.

The arising deformation disturbance (in activation) can be considered as an event of a higher scale in relation to those events that did not take place during a quiescence caused by the local hardening of the medium. The energy of the deformation disturbance must correspond to the sum of the energies of the adjourned events.

Local hardening of the medium and the preparation of the deformation disturbance on one of the spatial scales $L$ can be described with the fractional Poisson process, for which the average number of events in the time interval $t$ is given by the expression

$$
\bar{n}_{v}(t)=\frac{(\mu t)^{v}}{\Gamma(1+v)},
$$

where $\mu$ is the flow of events with the scale $L$ without hardening of the medium, $\Gamma(1+v)$-gammafunction, $0<v \leq 1[6,7]$. For $v=1$ we have the Poisson process with independent events.

The inverse $1 / v$ of the fractal parameter $v$ can be considered as a hardening parameter of the medium. Indeed, with its increasing (decreasing of parameter $v$ ), the event flow of $\bar{n}_{v}(t)$ slows down when $\mu t>1$, and the events deficit

$$
\bar{n}_{v}(t)-\bar{n}_{1}(t)
$$

causes an increase in stresses. And the time of accumulation of adjourned events until the moment when hardening of the medium is overcome will determine the energy of the deformation disturbance.

It is noteworthy that the parameter $v$ is responsible for the fractal dimension of the distribution of events on the time interval [8]. Thus, hardening of the medium (its rheological property) changes the fractality of the process (event statistics) that is realized as a delayed relaxation caused by memory effects that have resulted from the hardening.

The slowing down of the fractional process is related to the behavior of probability to keep the initial state of process $[6,7]$

$$
P_{0}(t)=E_{v}\left(-(\mu t)^{v}\right)
$$

this is the survival probability of the initial state, where $E_{v}(x)$ is the Mittag-Leffler function, the fractal generalization of the exponential, $0<v \leq 1$. However, not a function $E_{v}(x)$, but a complex function $E_{v}\left(-(\mu t)^{v}\right)$ is called a fractal exponential, it is the relaxation characteristic of the process. The properties of its complexity, behind which there are two effects, will be discussed below.

For small values of the parameter $v$, the relaxation of the initial state slows down, and the waiting times increase. The fading of pulsations is caused by this. The redistribution of probability is given by

$$
\Delta P_{0}(t)=E_{v}\left(-(\mu t)^{v}\right)-E_{1}(-\mu t)
$$

where $E_{1}(-\mu t)=e^{-\mu t}$.

Deviation of $P_{0}(t)$ from the usual exponential indicates the presence of aftereffects and memory, which are caused by the adjourned events. We'll considered the difference between memory and aftereffects in more detail.

When the hardening of the medium is overcome, the plastic deformation disturbance comes to replace of seismic quiescence. Disturbance has three phases: foreshocks, mainshock and aftershocks. In essence, these are the events that did not take place in seismic quiescence. The first and last phases of the disturbance are represented by a sparse packing of events and the mainshock is represented by dense one. Each event of the cluster affects on subsequent events, it is a aftereffect and at the 
same time, every event of the cluster is affected by previous events, it is a memory effect. The past impacts on the future through the present [8]. As a result, there is formed a chain of events so joint that the impacts of aftereffect and memory are difficult to separate, but below we will try to do it using different approximations.

Any event from the sequences of foreshocks, mainshock and aftershocks can be decomposed into the sum of background events with the scale of $L$. The total number of events with a scale $L$ in the plastic deformation disturbance should roughly respective to the number of adjourned events during the quiescence of the process. Such representation of the deformation disturbance allows us to estimate the disturbance energy and to establish a relation between the scales: foreshocks - the wave of consolidation of events of the selected scale level $L$, the mainshock is the realization of the disturbance at the highest scale level, and aftershocks - the wave of branching of the mainshock. There are the waves up and down on the scales, and in the space time presentation, we have converging and diverging waves to and from the center which is the mainshock.

Note that in the quiescence phase there is only a divergent wave from the adjourned events. This is a slow diffusion process, while in the deformation disturbance, the process is faster by 3-4 orders. The fast process is the wave in the mainshock and the fractioal diffusion in the foreshocks and aftershocks.

Thus, the evolution of the packing of events of the scale $L$ and the interaction of scales occurs in the deformation disturbance simultaneously as a result of consolidation and branching. Here we can note the analogy with turbulence in which the integration and fragmentation of vortices take place, an intermittency of regimes is observed. There is an analogy between the dislocation and vortex. We'll stay on their difference in spectra below.

The time form of the plastic deformation disturbance can be considered as a fractal analogue of the Berlage pulse with a variable packing density of events of scale $L$ and variable fractal dimension. For the disturbance model, we'll use various modifications of the fractal exponential $P_{0}(t)=E_{v}\left(-(\mu t)^{v}\right)$, which was apllied to describe the phase of the quiescence.

For the probability of the mainshock emergence we'll use the expression

$$
P_{t<t^{*}}(\tau)=E_{\alpha}\left(-(-\tilde{\mu} \tau)^{\alpha}\right)
$$

This is an increase (time reversal) fractal exponential, where $\tau$ is the time with the starting point from the moment of the start of the mainshock $t^{*}, \tau=t-t^{*}, \tau<0, \tilde{\mu}-$ is the average flow of events in the deformation disturbance, $0<\alpha \leq 1$. The probability of absence of the mainshock will be

$$
\tilde{P}_{t<t^{*}}(\tau)=1-P_{t<t^{*}}(\tau)
$$

Differentiating this expression, we'll obtain the distribution of the increasing flow of foreshocks, which can be considered as the inverse Omory law. This process will be the initial stage of the fractal pulse of Berlage.

For the probability of the mainshock preserving, we'll use the expression

$$
P_{t^{\prime}<t}\left(\tau^{\prime}\right)=E_{\beta}\left(-\left(\tilde{\mu} \tau^{\prime}\right)^{\beta}\right) \text {. }
$$

This is decreasing fractal exponential, where $\tau^{\prime}$ is the time with the starting point from the moment of the end of the mainshock $t^{\prime}, \tau^{\prime}=t-t^{\prime}, \tau^{\prime}>0$, the parameter $\tilde{\mu}-$ is the average flow of events in the deformation disturbance, $0<\beta \leq 1$. The probability of the mainshock not preserving will be

$$
\tilde{P}_{t^{\prime}<t}\left(\tau^{\prime}\right)=1-P_{t^{\prime}<t}\left(\tau^{\prime}\right) .
$$

Differentiating this expression, we'll obtain the distribution of the decreasing flow of aftershocks, i.e. the Omory law, that can be considered as the attenuation stage of the fractal Berlage pulse. 
To sew together the two obtained fractal expressions, we'll take into account that the flow of events in the phase of mainshock is tightly packed and constant, and the intencity of fractal flows can not exceed the tight packing. In the foreshock phase, the event package increase to the maximum possible, in the mainshock it persists, and in the aftershocks it decreases. Fractal parameters $\alpha$ and $\beta$ are determined by the properties of the medium, its capacity to consolidate and to branch events. The average flow of events $\tilde{\mu}$ in the plastic deformation disturbance is determined by the phase of the quiescence, which is characterized by the parameters $\mu, v$ and the time of quiescence. The time scales $\mu$ and $\tilde{\mu}$ can differ by 3-4 orders, since the perturbation is short-lived.

The phase of the foreshocks in the disturbance is usually very fast. This is a short front of the fractal Berlage pulse. So short in the compearison with the aftershocks phase that it can be neglected in the first approximation and only aftershocks as the branching of the mainshock can be considered (ETAS model) [5]. However, for the earthquakes forecasting, it's just the foreshock phase and the preceding quiescence stage are of interest. Using the length of quiescence time, it is possible to estimate the energy of the stress discharge and the phase of the foreshocks predicts the rate of the plastic deformation disturbance, that gives the time of the short-term forecast. In view of this, a new theory is needed.

\section{Comparison with the ETAS model}

It remains to show that our result corresponds to the ETAS model, if we neglect the effects of memory. For the probability of the initial state survivaling, the ETAS model gives [5]

$$
P(\tau)=e^{-\nu \tau-b(v \tau)^{1-\theta}} .
$$

Proceeding from the form of the exponential of this expression, we have two flows of events. The first of them corresponds to a Poisson process with a constant flow, and the second - to a process with a flow of events decreasing in time on power law. The second flow takes into account aftershocks as branching of the events of the first one. Neglecting the weaker first flow and differentiating the expression $1-e^{-b(v \tau)^{1-\theta}}$ for the transition probability from the initial state to any other, we obtain the Weibull distribution for the aftershocks waiting times.

For the aftershock phase, our result gives expression

$$
P_{t^{\prime}<t}\left(\tau^{\prime}\right)=E_{\beta}\left(-\left(\tilde{\mu} \tau^{\prime}\right)^{\beta}\right)
$$

Replacing in it the fractal exponential on expanded one

$$
P_{t^{\prime}<t}\left(\tau^{\prime}\right) \simeq e^{-\left(\tilde{\mu} \tau^{\prime}\right)^{\beta}},
$$

we get the result of the ETAS model, which agrees well with seismic data [5], however, more subtle effects are possible, which will be discussed below.

The differentiation of the expression

$$
1-P_{t^{\prime}<t}\left(\tau^{\prime}\right) \simeq 1-e^{-\left(\tilde{\mu} \tau^{\prime}\right)^{\beta}},
$$

which is the probability of transition from the initial state to any other, gives, as in the ETAS model, the Weibull distribution for the aftershocks waiting times. The values $0<\beta<1$ correspond to a decreasing flow of events (aftershocks), Omori's law. The value $\beta=1$ corresponds to a constant flow of events - the Poisson process. In solid state physics parameter $\beta$ is called the Weibull index.

From the comparison of the fractal approach with the ETAS model, we can conclude that the power law argument $(\mu t)^{v}$ of the fractal exponential $E_{v}\left(-(\mu t)^{v}\right)$ is responsible for the branching processes 
(aftereffects that determine the fractal slowing of the flow), and the function $E_{v}(x)$ itself describes the effects of memory. Both $(\mu t)^{v}$ and $E_{v}(x)$ give the delayed relaxation.

There are no memory effects in the ETAS model, and replacing the expression $E_{v}(x) \rightarrow e^{-x}$ changes the relaxation essentially. Thus, the fractal approach takes into account the history of the process and opens up more wider possibilities for describing the properties of the medium.

For example, to simulate the relaxation we can use a dependence $\tilde{P}_{0}(t)=E_{\tilde{v}}\left(-(\mu t)^{v}\right)$ with different exponents of branching $v$ and memory $\tilde{v}$. This approach corresponds to a fractional Poisson process with a time varying on power law flow of events. Wherein the number of fractional characteristics of the medium is doubled.

\section{The physical significance of the exponent $b=3 / 2$}

We note that for foreshocks the power law argument of the fractal exponential is responsible for consolidation processes, but not the branching. The process of reverse diffusion (consolidation) has the opposite direction in a nonequilibrium medium. In the fault, two processes with opposite directions in the space of the scales are presented simultaneously. They interact in the system of opposing waves, forming a fractal wave of destruction (a pair of kink and antikink), which interacts in a complex way with the nonequilibrium medium, obtaining the energy of elastic deformations to produce fractures and generate elastic and electromagnetic oscillations. The model of the complex of fractal wave processes can be the subject of the following paper, but here we make only a few remarks.

In the coordinate system associated with the wave of fracture, the dislocation process can be represented as an oscillator. This is a well-known description of dislocation changes using the sine-Gordon equation. Fractal properties of the process are introduced to describe the interaction of the fracture wave with the medium. The period of the oscillations is determined by the time $\tau_{L}$ of formation of one dislocation of scale $L$ in the fault. And the number of oscillations $n_{R}$ is the ratio $n_{R}=\tau_{R} / \tau_{L}$, where $\tau_{R}$ is the time of formation of the discontinuity, the parameter $n_{R}$ plays the role of a topological quantum number. Similar numbers can be introduced for foreshocks and aftershocks, $\breve{n}_{R}$ and $\hat{n}_{R}$. Naturally, the sum of all the quantum numbers (the number of events of scale $L$ in the cluster) must correspond to the deficit of events in the period of quiescence:

$$
S_{D}=\check{n}_{R}+n_{R}+\hat{n}_{R}
$$

i.e. the quantum number of the deformation disturbance. This value, which is determined by the properties of the medium, characterizes the energy of the plastic deformation disturbance and the ratio of the scale levels.

The fractal structure of the deformation disturbance is opened by a wavelet transformation that is an expansion in the terms of the basis impulse functions, the form of which is consistent with the form of the disturbance under consideration. Such expansion was called the sparse approximation $[9,10]$, for which Berlage pulses are used as the basis functions. It is obvious that the number of wavelet summands can be compared to the quantum number of the deformation disturbance $S_{D}$ (13). Each of the wavelet summands gives a detailed information how the fault is formed.

An analogy with quantum mechanics arises here, using which the structure of elementary particles can be represented as a deformation disturbance.

The formation of the fault can be regarded as a Poisson process, simple or fractal, and hence for a faults distribution on scales $p(L)$, which corresponds to the Gutenberg-Richter law, we will have a stable or fractional stable distribution. What are the differences between these distributions, see [8]. For us, the most interesting are fractional stable distributions, in which, as was shown above, the effects of branching and memory are taken into account. 
In the formation of a fault, phased (coherent) elastic and electromagnetic oscillations arise [11]. This is due to the pair of forces and an element of current appear simultaneously in the dislocation, which serve as sources of two types of oscillations. And the fault can be represented as a flow of dislocations. It is interesting to find the relation between the energy and the spatial scale of the fault (and the perturbation as a whole), which until now is known only from observations. Let us turn again to an analogy with quantum mechanics.

Using the sine-Gordon equation, the formation of a discontinuity (fault) can be represented as the motion of a wave on a periodic potential with the addition of fracture energy and elastic deformations. Here we do not consider the loss of radiation. The Hamiltonian of these oscillations will have the form

$$
E^{2}=m^{2}(L)+p^{2}-\sigma^{2} L^{2}+\varepsilon k L^{3},
$$

where $E$ is the oscillation energy operator, $m^{2}(L)$ is the periodic potential (for the Gordon equation), $p$ is the impulse operator, $\sigma^{2} L^{2}$ is the energy of fractures, $\sigma^{2}$ is the specific energy of destruction, $\varepsilon k L^{3}$ is the energy of elastic deformations, $\varepsilon$ is relative deformation (their critical level is supported by the work of external forces ), $k$ is the shear modulus.

A wave of unlimited destruction is possible if there is satisfied the Griffith condition $L>\sigma^{2} /(\varepsilon k)$. For $L \rightarrow \infty(p \sim 1 / L)$ we obtain the asymptotic expression $E^{2} \simeq \varepsilon k L^{3}$, which gives the relation between the energy of elastic oscillations and the scale of the distubance $E \simeq \sqrt{\varepsilon k} L^{3 / 2}$. This expression is used to conversion the magnitude to the logarithm of the event energy, the exponent $b=3 / 2$ ( $b$-value in the expression $\lg E=a+b \lg L$, where $\lg L=M$ is the magnitude). Until now, the value $b=3 / 2$ was known only from experimental data, and the physical significance of the parameter $a=\sqrt{\varepsilon k}$ was not discussed till now. It should be specially noted that the value $\varepsilon$ is a characteristic of the state of the medium and has the physical significance of critical elastic relative deformations.

\section{Discussion and conclusions}

Turbulent velocity pulsations satisfy the following relation $v \sim l^{1 / 3}$, and the energy $v^{2} \sim l^{2 / 3}$, where $l$ is the scale of pulsations. Turbulence has much in common with deformation disturbance (destruction), but their power law pulsation spectra differ in the exponents, which is due to differences in the initial dynamical equations.

Deformation disturbance have much in common with electrical breakdown and lightning discharges, with acoustic and electromagnetic fluctuations in the plasma. The developed statistical model will help to look from another point of view at the suddenness of precipitations from the radiation belts, the features of the aurora borealis and the nature of globe lightning. And the analogy with quantum mechanics opens new possibilities in studies of fractal dynamics of elementary particles and cosmic rays.

Plastic deformations have much in common with epidemics, see ETAS model [5], the prototype of which was the Markov-Poya process. Its fractal generalization will allow to take into account the effects of memory in the diseases.

The multiplicity of analogies between different physical processes indicates that they belong to the class of critical phenomena.

Plastic deformation disturbances develop on orders faster than the period of their preparation. Therefore, the disturbances are sources of abnormal increased seismoacoustic and electromagnetic emission, which acquires statistical characteristics of the related dislocational changes in clusters, that allows using anomalies in signals of emission to diagnose the activation of deformation processes. The intensity of emission in the background and during the seismic quiescence period is significantly lower than in activation phase. And the differences between the background and the quiescence period can 
be insignificant, therefore long observations and their comparative analysis will be required to identify a shortage (deficit) of events that will lead to a relaxation of stresses.

Emission of plastic deformation disturbance plays a special role in the processes of lithosphereionospheric interaction precisely because its intensity far exceeds the emission of the background regime. Ionospheric effects are observed using ionosonde, GPS technologies and lidars in the variations of the ionosphere parameters, the precipitation of charged particles from the radiation layers and the formation of light scattering layers in the ionosphere by excited atoms and ions. The comparable effects in the ionosphere can also be created by lightning discharges.

A fractal model of plastic deformation disturbance and associated seismoacoustic and electromagnetic emission is proposed.

The fractal model includes branching and memory effects. ETAS model - only branching.

The fluctuation spectra of the deformation perturbation and turbulence are differ on a reason of the differences in their dynamic equations.

A statistical justification of the Gutenberg-Richter law is provided using stable distributions for the background regime and fractional stable one for the phases of quiescence and disturbance. For a more detailed description, it is proposed to use the fractinalal Poisson process with flow a time varying on power law.

The topological quantum number arising in the representation of a deformation disturbance in the form of a fractal pulse Berlage is determined by the deficit of events at the stage of preparation of the deformation disturbance and when processing signal it is obtained with using a sparse approximation of the disturbance.

The topological quantum number defines the relation between scale levels and can be used as the logarithm base in scaling.

A dynamic relation was determined between the energy and the spatial scale of the deformation disturbance (dislocation, seismic event). Thus, it is given the justification for the transformation from the magnitudes to the logarithm of the event energy.

\section{References}

[1] B.M. Shevtsov, R.N. Sagitova, Doklady Earth Sciences, 426(2), 642-644 (2009)

[2] B.M. Shevtsov, R.N. Sagitova, Volcanology and Seismology, 2, 56-66 (2012)

[3] A.S. Perezhogin, B.M. Shevtsov, R.N. Sagitova, G.M. Vodinchar, Matem. Mod., 19(11), 59-64 (2007)

[4] A.S. Perezhogin, B.M. Shevtsov, Computational technologies, 14(3), $48-57$ (2009)

[5] A. Saichev, D. Sornette, J. Geophys. Res. 112 (2007)

[6] D.O. Cahoy, V.V. Uhaikin, W.A. Woyczyski, Journal of Statistical Planning and Inference, 140, 3106-3120 (2010)

[7] N. Laskin, Communications in Nonlinear Science and Numerical Simulation, 8, 201-213 (2003)

[8] V.V. Uchaikin, Uspekhi Fizicheskikh Nauk (UFN), 173, 847-876 (2003)

[9] Y.V. Marapulets, A.B. Tristanov, B.M. Shevtsov, Akusticheskij zhurnal, 60(4), 427-435 (2014)

[10] Y.V. Marapulets, A.B. Tristanov, B.M. Shevtsov, Doklady Earth Sciences, 456(2), 705-708 (2014)

[11] V.N. Uvarov, Geophysical Journal, 38(6), 180-185 (2016) 